\title{
Biologically active form of vitamin B1 in human peritoneal effluent
}

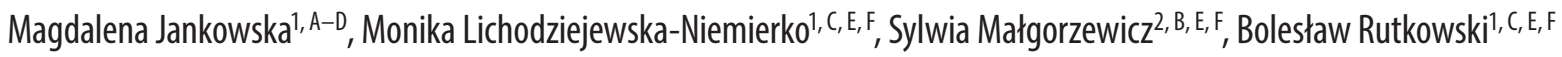 \\ ${ }^{1}$ Department of Nephrology, Transplantology and Internal Medicine, Medical University of Gdańsk, Poland \\ ${ }^{2}$ Department of Clinical Nutrition and Dietetics, Medical University of Gdańsk, Poland \\ A - research concept and design; $\mathrm{B}$ - collection and/or assembly of data; C - data analysis and interpretation; \\ $D$ - writing the article; $E$ - critical revision of the article; $F$ - final approval of the article
}

\author{
Address for correspondence \\ Magdalena Jankowska \\ E-mail:maja@gumed.edu.pl \\ Funding sources \\ None declared

\section{Conflict of interest} \\ None declared

\section{Acknowledgments} \\ We are grateful to Dr. Marcin Marszałł, \\ who carried out the extensive laboratory \\ work in the current study.
}

Received on August 23, 2016

Reviewed on November 4, 2016

Accepted on January 30, 2017

\section{Abstract}

Background. Supplementation with vitamin B1 protects the peritoneal membrane from inflammatory and oxidative insults and preserves residual kidney function in rat models of peritoneal dialysis (PD). It is assumed that an active form of vitamin B1, thiamin diphosphate (ThDP), is responsible for this protective effect. However, it has never been shown whether ThDP, a compound known not to cross cellular membranes, is actually detectable in human peritoneal effluent.

Objectives. This study was designed to investigate the concentration, appearance rate, and daily loss of ThDP in the peritoneal effluent of patients treated with PD.

Material and methods. We performed 24-hour effluent collection as well as the peritoneal equilibration test (PET) and analyzed the relation between the transport characteristics of the peritoneal membrane and appearance rate of ThDP in a cohort of $26 \mathrm{PD}$ patients.

Results. ThDP was detectable in peritoneal effluent in humans. ThDP appearance rate was independent of the transport characteristic of peritoneal membrane, and was not associated with peritoneal transport of other small solutes.

Conclusions. We conclude that ThDP can be found in detectable concentrations in the peritoneal effluent in humans and is transported through the peritoneal membrane in a pattern independent of other small solutes. Our finding opens novel opportunities in further research on the protection of peritoneal membrane in humans.

Key words: peritoneal dialysis, thiamine, micronutrient

DOI

10.17219/acem/68722

\section{Copyright}

Copyright by Author(s)

This is an article distributed under the terms of the

Creative Commons Attribution Non-Commercial License

(http://creativecommons.org/licenses/by-nc-nd/4.0/) 


\section{Background}

Thiamin, also known as vitamin B1, is an essential nutrient for humans. Whereas it is synthesized by many plants and microorganisms, all mammals depend on its consumption with diet. Besides being a potent cofactor for numerous enzymatic activities essential in energy metabolism pathways, thiamin is involved in brain functioning and interneuronal communication. ${ }^{1}$ Also, it plays a role in the regulation and activation of the immune system. Interestingly, via suppressing activation of necrosis factor $N_{k} B$, thiamin inhibits a release of inflammatory markers by macrophages. ${ }^{2}$ Vitamin B1 has also been regarded to constitute anti-inflammatory factor and regulate the expression of inflammatory agents. ${ }^{3}$ Thiamin has received increased interest recently, since the discovery that it is able to reduce peritoneal AGE accumulation, fibrosis, neovascularization, and inflammation in uremic rats. Also, it preserves the remnant kidney function and has an impact on residual renal function in peritoneal dialysis (PD). ${ }^{4}$ These protective effects are assumed to occur due to the action of thiamin diphosphate (ThDP), a biologically active derivative of vitamin B1. It is a product of an intracellular reaction involving thiamin phosphokinase activity. ${ }^{1}$ Interestingly, unlike thiamin monophosphate or free thiamin cation, ThDP is believed not to cross cellular membranes. ${ }^{1}$ Hence, despite the beneficial effect of ThDP described in a PD model in rats, it is not known whether this compound can be detected in human peritoneal effluent.

Taking into account the high prevalence of inadequate vitamin B1 status, which may lead to neuropathy, autonomic dysfunction, impaired immune system reactivity, and enhanced inflammatory response, the scarcity of evidence concerning peritoneal thiamin losses may be surprising. Only few studies have assessed vitamin B1 status in PD patients. ${ }^{5-10}$ To the best of our knowledge, only 1 measured vitamin B1 peritoneal losses. ${ }^{5}$ However, ThDP losses have not been assessed and reported yet. Consequently, there is no data on the factors that may contribute to the magnitude of such losses, and the relation of ThDP appearance rate to the transport characteristics of the peritoneal membrane has never been addressed.

The peritoneal equilibration test (PET) is a clinical tool to assess the peritoneal membrane characteristics of individual patients in order to provide an appropriate PD prescription. The PET can be used to estimate transport properties of the peritoneal membrane. Assessment of ThDP in dialysis effluent during the PET makes it possible to analyze active thiamin compound appearance in the effluent under standardized and reproducible conditions. Also, it makes it possible to analyze the relationship between ThDP loss and the transport characteristics of the peritoneal membrane.

The aim of our research was to measure ThDP concentration in the peritoneal effluent, to assess ThDP losses related to $\mathrm{PD}$, and to predict the pattern of ThDP trans- port, as compared to the transport of blood urea nitrogen (BUN), creatinine, and glucose in PD patients.

\section{Methods}

Written consent was obtained from all participants before entering the study, and the local ethics committee approved the protocol. In this cross-sectional study, we recruited 26 adult participants. They comprised 16 patients treated with continuous ambulatory peritoneal dialysis (CAPD) and 10 patients treated with automated peritoneal dialysis (APD). Patients with an episode of peritonitis in the preceding 30 days, with PD catheter malfunction, ultrafiltration failure, uncontrolled blood glucose, active infection, or the ones clinically overhydrated or dehydrated were excluded from the study. On the day of the PET performance, all patients underwent estimation of dialysis adequacy $(\mathrm{KT} / \mathrm{V})$, normalized protein catabolic rate (nPCR) and calculation of glomerular filtration rate (GFR) and peritoneal clearances based on 24-hour collections of urine and dialyzate. We measured ThDP, creatinine, BUN and protein levels in a 24-hour effluent collection. The PET was performed according to standard procedure (2-liter bag of $2.3 \%$ glucose solution, effluent sampling at $0 \mathrm{~h}, 2 \mathrm{~h}$, and $4 \mathrm{~h})$. ThDP was measured in dialysis effluent at $2 \mathrm{~h}$ and $4 \mathrm{~h}$. In 8 patients, a blood sample for ThDP analysis in plasma was also taken at $2 \mathrm{~h}$. Plasma was separated after centrifugation at 4500 RPM and stored with samples of dialysis effluents in $-80^{\circ} \mathrm{C}$ for further processing.

We also analyzed demographic (age, sex, body mass index (BMI), time on dialysis) and clinical (diabetes mellitus (DM) comorbidity, PD modality, ultrafiltration rate (UF), residual renal function (RRF), glomerular filtration rate, peritoneal transport characteristics, peritoneal clearance, normalized protein catabolic rate (nPCR), weekly $\mathrm{KT} / \mathrm{V}$, hemoglobin $(\mathrm{Hb})$, hematocrit $(\mathrm{Htc})$, and neutrophil to lymphocyte ratio (N/L)) indices, as predicted contributors that may influence ThDP concentration.

Total body water was estimated according to Watson's formula, and nPCR was calculated using Bergstrom's formula. ${ }^{11,12}$ All laboratory measures were performed with an autoanalyzer (Modular Roche, Roche Diagnostics, Indianapolis, USA), using standard methods as follows: BUN with the spectrophotometric method, creatinine with the colorimetric kinetic test, glucose with the spectrophotometric hexokinase method, and protein with the colorimetric method. Complete blood count was performed with flow cytometry (Sysmex XE 2100D, Sysmex Corp., Kobe, Japan).

ThDP levels in effluent and plasma were detected using high performance liquid chromatography (HPLC Dionex equipped with C-18 reversed phase column Hypersil Gold, Dionex Corp., Sunnyvale, USA) with coupled detection - spectrophotometric (detector UV 340S Dionex) and coulometric (detector Coulochem II model 5019 ESA, 
Thermo Fisher Scientific, Waltham, USA). The calibration curves proved to be linear; the method is characterized by a precision of $2.9 \%$. The minimum limit of quantification (LOQ) was equal to $8.7 \mathrm{ng} / \mathrm{mL}$; the minimum limit of detection (LOD) was equal to $2.9 \mathrm{mg} / \mathrm{mL}$.

\section{Statistics}

Distributions of all data were tested according to the Shapiro-Wilk test. Variables are presented as medians (95\% CI) and ranges, when appropriate. Multiple regression was performed in order to find associations between ThDP concentration and appearance rates and

Table 1. Clinical and biochemical characteristics of participants

\begin{tabular}{|l|c|}
\hline \multicolumn{1}{|c|}{ Characteristic } & $\begin{array}{c}\text { Median }(95 \% \mathrm{Cl}) \\
\mathrm{n}=26\end{array}$ \\
\hline Age (years) & $56(50-59)$ \\
\hline Gender (M/F) & $12 / 14$ \\
\hline No. of diabetics & 5 \\
\hline Time on PD (months) & $18(12-46)$ \\
\hline PD modality (APD/CAPD) & $(10 / 16)$ \\
\hline BMI (kg/m²) & $25.3(24.1-27.9)$ \\
\hline Residual renal function: GFR (mL/min/1.73 m²) & $2.9(2.8-6.1)$ \\
\hline Urine output (mL/24 h) & $900(676-1296)$ \\
\hline Plasma creatinine (mg/dL) & $7.8(7.1-9.7)$ \\
\hline Plasma BUN (mg/dL) & $48.5(44.8-60.0)$ \\
\hline Plasma glucose (mg/dL) & $92(86-106)$ \\
\hline Hemoglobin (g/L) & $10.8(10.2-11.1)$ \\
\hline Hematocrit (\%) & $33(31-34)$ \\
\hline Neutrocytes/lymphocytes ratio & $2.8(2.4-3.6)$ \\
\hline CrCl (mL/min/week) & $68(69-94)$ \\
\hline pCrCl (mL/min/week) & $39.5(30.6-41.3)$ \\
\hline Kt/V (weekly) & $2.1(2.05-2.73)$ \\
\hline nPCR (g/kg/24 h) & $1.20(1.10-1.40)$ \\
\hline Protein peritoneal loss (g/24 h) & $0.5(0.4-0.7)$ \\
\hline Ultrafiltration at 4 h (mL) & $450(368-493)$ \\
\hline No. of patients receiving thiamine supplements & 15 \\
\hline (6) daily) & \\
\hline
\end{tabular}

potential contributors. Univariate correlations between the variables were calculated using the Spearman correlation coefficient. Statistical significance was considered at $\mathrm{p}<0.05$. Data analysis was accomplished using STATISTICA v. 12 PL (StatSoft, Inc., Tulsa, USA).

\section{Results}

The characteristics of the study group are shown in Table 1. With regard to the PET results, the mean value of $\mathrm{D} / \mathrm{P}$ (dialyzate-to-plasma ratio) of creatinine at $240 \mathrm{~min}$ was $0.59 \pm 0.1$. Four participants were classified as fast, 14 as average fast/high, 7 as average slow/ low, and 1 as slow transporter. Figure 1 depicts ThDP appearance rate at $240 \mathrm{~min}$ of the PET and ThDP loss in 24-hour effluent collection in individual participants. Both parameters varied markedly interindividually. Nevertheless, the appearance rate and daily losses of ThDP were significantly correlated (Spearman $r=0.702$; $\mathrm{p}<0.05)$. Also, a relationship was found between daily loss of ThDP and its concentration in effluent at the $2^{\text {nd }}$ and $4^{\text {th }}$ hour of the PET (Fig. 2).

The median value of ThDP daily loss is shown in Table 2. Table 2 also shows ThDP concentrations in 24hour effluent collection, and in samples at $2 \mathrm{~h}$ and $4 \mathrm{~h}$ of the PET. In 8 patients, plasma concentrations of ThDP were also measured. In all cases, the plasma values were low, close to the lower limit of the reference range for humans.

In multivariate regression, supplementation with thiamin hydrochloride, the modality of PD treatment (CAPD vs APD), diabetic status or anuria were not associated with ThDP appearance rate and losses. ThDP losses, dialyzate concentration and appearance rates could also not be explained by age, $\mathrm{BMI}$, time on $\mathrm{PD}$, ultrafiltration rates, peritoneal protein loss, nPCR, weekly $\mathrm{KT} / \mathrm{V}$, total week clearances of urea, and creatinine or by residual GFR.

ThDP effluent concentrations and appearance rates were not associated with plasma ThDP, as well as plasma and effluent concentrations and appearance rates of BUN and creatinine. There was no relationship between ThDP and plasma or effluent glucose concentrations. Also, peritoneal membrane characteristics, as classified in the PET, did not influenced daily ThDP losses.

In univariate analysis, we found weak associations between ThDP effluent concentrations at $4 \mathrm{~h}$ of the PET and N/L index ( $\mathrm{r}=0.397 ; \mathrm{p}<0.05)$, peritoneal urea and creatinine clearances $(r=0.487$ and $r=0.429$, respectively; $\mathrm{p}<0.05)$ and patients' weight and height $(r=-0.460$ and $r=-0.499$;
Table 2. Thiamin diphosphate in peritoneal effluent and plasma

\begin{tabular}{|l|c|c|}
\multicolumn{1}{|c|}{ Variable } & $\begin{array}{c}\text { Median }(95 \% \mathrm{Cl}) \\
\mathrm{n}=26\end{array}$ & $\begin{array}{c}\text { Range } \\
\mathrm{n}=26\end{array}$ \\
\hline Peritoneal loss in $24 \mathrm{~h}(\mu \mathrm{g})$ & $92.6(55.4-351.6)$ & $11.9-1546.7$ \\
\hline Appearance rate in effluentafter $240 \mathrm{~min}(\mathrm{ng} / \mathrm{min})$ & $103.1(86.7-124.6)$ & $38.3-176.5$ \\
\hline Concentration in 24-hour effluent collection $(\mathrm{ng} / \mathrm{mL})$ & $91.0(58.3-330.1)$ & $168-1381$ \\
\hline Concentration in effluent at the $2^{\text {nd }}$ hour of the PET $(\mathrm{ng} / \mathrm{mL})$ & $80(70.8-115.9)$ & $16-222$ \\
\hline Concentration in effluent at the $4^{\text {th }}$ hour of the PET $(\mathrm{ng} / \mathrm{mL})$ & $93(85.5-123.9)$ & $38-181$ \\
\hline Concentration in plasma $(\mathrm{ng} / \mathrm{mL})$ & $17.2(14.7-19.3)^{*}$ & $13.2-22.2^{*}$ \\
\hline
\end{tabular}

${ }^{*} \mathrm{n}=8$.

$$
\mathrm{p}<0.05) \text {. }
$$



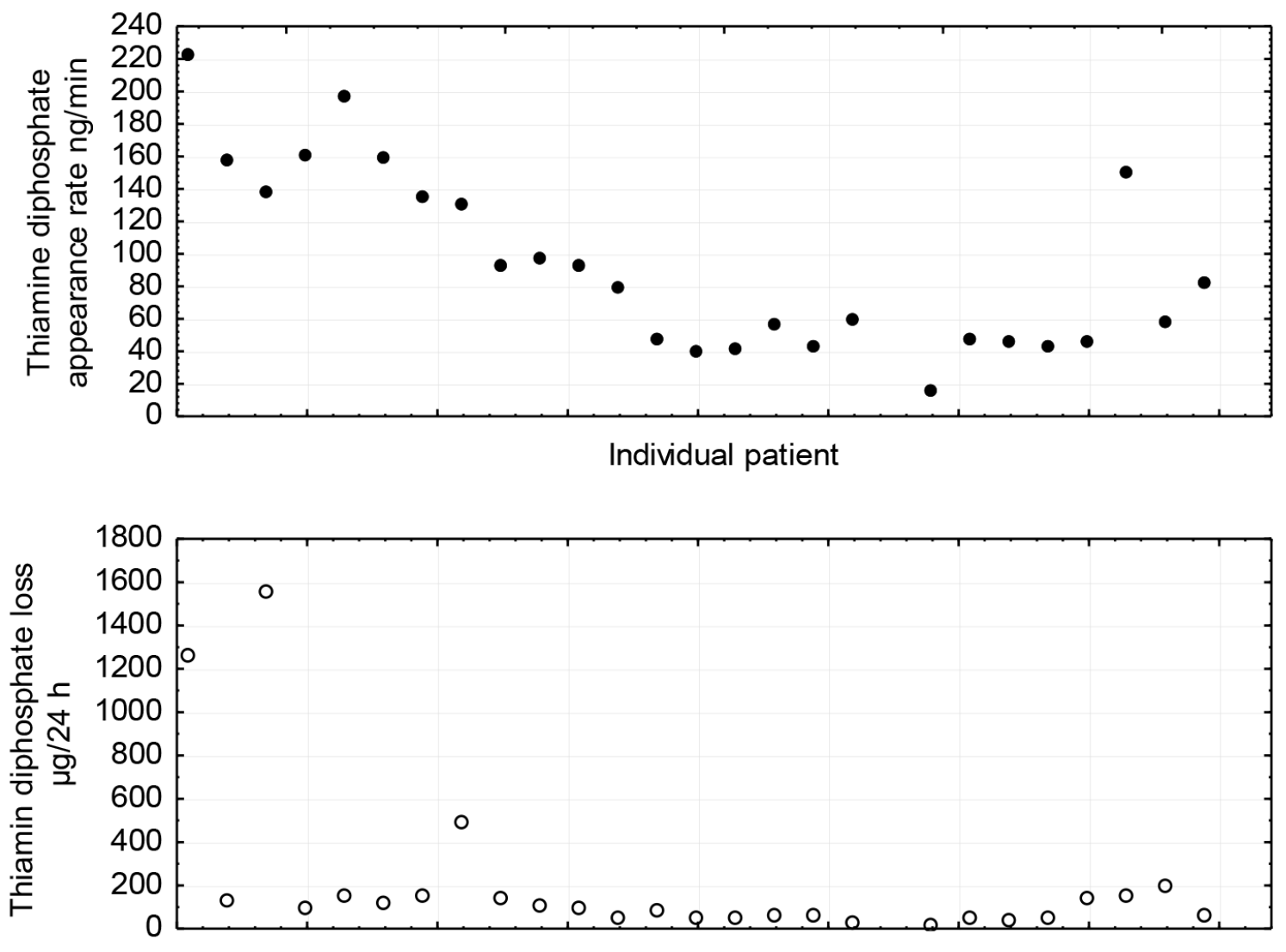

Individual patient

Fig. 1. Appearance rate of thiamin diphosphate (ThDP) at $240 \mathrm{~min}$ of the peritoneal equilibration test (PET) and daily dialyzate losses of ThDP in all individual cases $(n=26)$

\section{Discussion}

Peritoneal losses and concentration of ThDP, a biologically active form of thiamine, in human dialyzate, have not been studied so far - our study aimed to fill this gap in knowledge. In view of the recent findings of the benefits of thiamine supplementation, which seems to protect the peritoneal membrane from inflammatory and oxidative insults, knowledge of ThDP may have important clinical implications. The PET allowed us to analyze ThDP losses under standardized and reproducible conditions. The most important finding of the presented study has been an unequivocal identification of ThDP in dialysis effluents. Thus, we have not only confirmed the previous findings of Boeschoten et al. that thiamine may be lost by this route, but we have also shown that the thiamine active form appears in the peritoneal effluent. ${ }^{5}$ ThDP losses, although modest in most cases, may exceed daily recommended intake (DRI) for vitamin B1, as is depicted in Fig. 1. Thiamine loss seems to be independent of the transport characteristics of the peritoneal membrane, but we have found significant positive associations between effluent ThDP concentrations and peritoneal clearances of urea and creatinine. Effluent BUN concentrations also correlated with those of ThDP in the $4^{\text {th }}$ hour of the PET. Thus, thiamine transport follows to some extent the pattern of the transport of small solutes.

Plasma ThDP levels proved to be below the reference range for the method used $(20-50 \mathrm{ng} / \mathrm{mL})$, in most analyzed cases. Interestingly, plasma thiamine levels are known to respond to carbohydrate intake in humans. ${ }^{13}$ It is plausible that glucose absorption from $2.3 \%$ dialytic solution is responsible for the low plasma ThDP in the $2^{\text {nd }}$ hour of the PET. However, we have not found any correlation between effluent glucose concentration and plasma ThDP to support such a hypothesis.

We found a higher concentration of ThDP in the 24-hour effluent collection than the concentration in plasma. Hence, some mechanism of active transport or local ThDP synthesis must be responsible for this phenomenon. Mean ThDP D/P is well above 1, indicating other than simple gradient diffusion ways of ThDP dialyzate appearance. Neutrocyte/lymphocyte (N/L) ratio, reflecting systemic inflammatory status, was a parameter significantly associated with thiamine D/P. However, it is unclear whether it reflects the changed transport characteristics of the peritoneal membrane or rather local increased turnover of ThDP in the peritoneal cavity. This is an interesting finding that needs further investigation. 


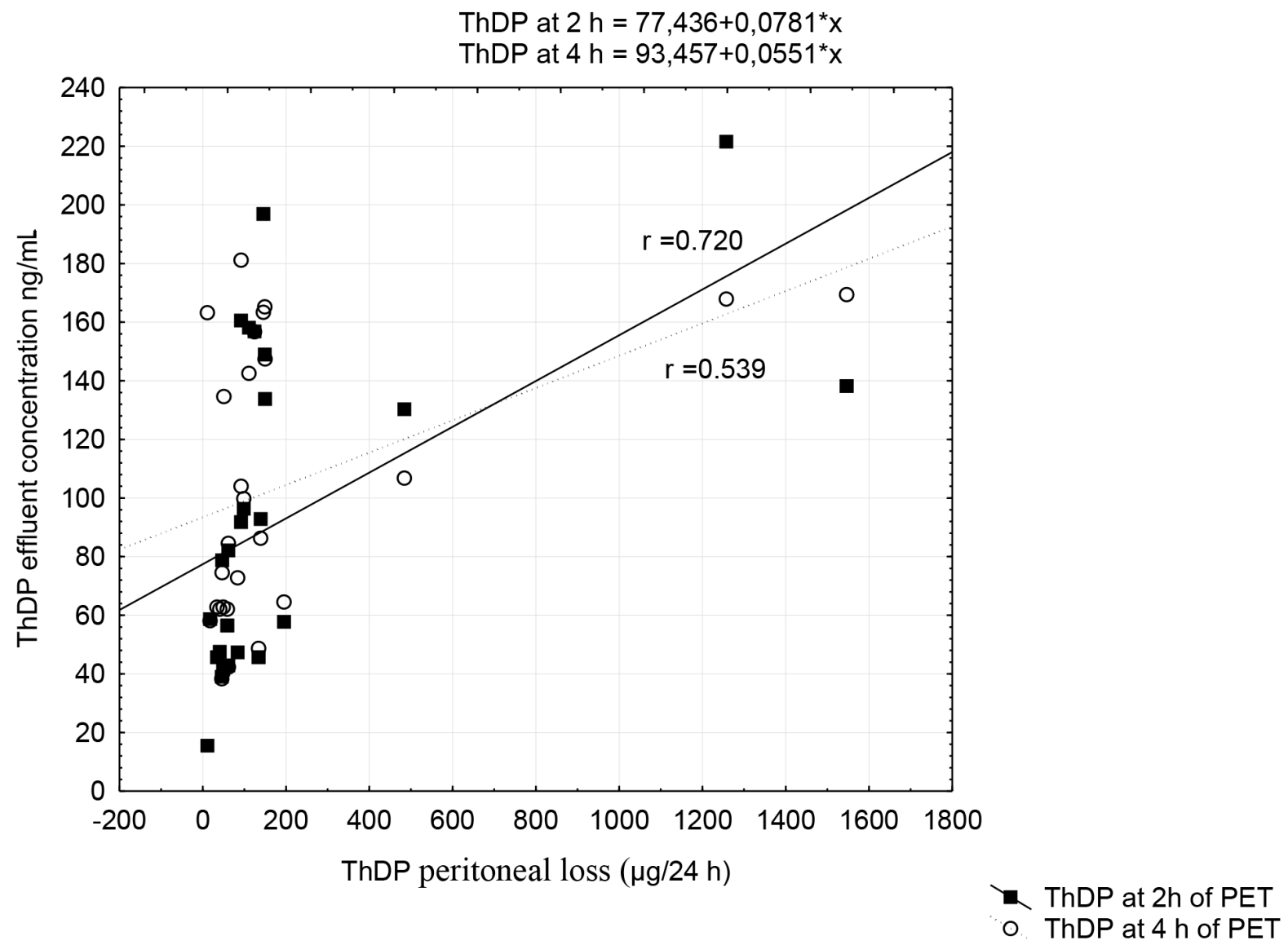

Fig. 2. Peritoneal loss and effluent concentrations of thiamin diphosphate(ThPD) at $2 \mathrm{~h}$ and $4 \mathrm{~h}$ of the peritoneal equilibration test (PET)

The change of glucose concentration in effluent has not influenced ThDP levels. Thus, convection seems to have little impact on ThDP peritoneal losses.

Unexpectedly, supplementation with vitamin B1 seems to have no impact on peritoneal ThDP losses. Supplement derived thiamine, which occurs in unphosphorylated compound, can be easily lost with urine. ${ }^{14}$ Taking into account the decreased vitamin B1 ingestion due to dietary restrictions, impaired absorption, and dialysis-related losses shown in this study, the need for thiamine supplements in PD patients should be widely recognized. ${ }^{15,16}$

In conclusion, we have shown that the active, phosphorylated form of thiamine is present in the dialyzate effluents of PD patients. We have also shown that ThDP appearance in dialyzate is independent of the transport characteristics of the peritoneal membrane. Our findings offer novel perspectives for future research on the protection of the peritoneal membrane in humans.

\section{References}

1. Manzetti S, Zhang J, van der Spoel D. Thiamin function, metabolism, uptake, and transport. Biochemistry. 2014;53(5):821-835.

2. Yadav UC, Kalariya NM, Srivastava SK, Ramana KV. Protective role of benfotiamine, a fat-soluble vitamin B1 analogue, in lipopolysaccharide-induced cytotoxic signals in murine macrophages. Free Radic Biol Med. 2010;48(10):1423-1434.
3. Shoeb M, Ramana KV. Anti-inflammatory effects of benfotiamine are mediated through the regulation of the arachidonic acid pathway in macrophages. Free Radic Biol Med. 2012;52(1):182-190.

4. Kihm LP, Müller-Krebs S, Klein J, et al. Benfotiamine protects against peritoneal and kidney damage in peritoneal dialysis. J Am Soc Nephrol. 201122(5):914-926.

5. Boeschoten EW, Schrijver J, Krediet RT, Schreurs WH, Arisz L. Deficiencies of vitamins in CAPD patients: The effect of supplementation. Nephrol Dial Transplant. 1988;3(2):187-193.

6. Blumberg A, Hanck A, Sander G. Vitamin nutrition in patients on continuous ambulatory peritoneal dialysis (CAPD). Clin Nephrol. 1983;20(5):244-250.

7. Mydlík M, Derzsiová K, Válek A, Szabó T, Dandár V, Takác M. Vitamins and continuous ambulatory peritoneal dialysis (CAPD). Int Urol Nephrol. 1985;17(3):281-286.

8. Mydlik M, Derzsiová K. Erythrocyte vitamins B1, B2 and B6 and erythropoietin. Am J Nephrol. 1993;13(6):464-466.

9. Henderson IS, Leung ACT, Shenkin A. Vitamin status in continuous ambulatory peritoneal dialysis. Perit Dial Bull. 1984;4:143-145.

10. Skoupy S, Födinger $M$, Veitl $M$, et al. Riboflavin is a determinant of total homocysteine plasma concentrations in end-stage renal disease patients. J Am Soc Nephrol. 2002;13(5):1331-1337.

11. Watson PE, Watson ID, Batt RD. Total body water volumes for adult males and females estimated from simple anthropometric measurements. Am J Clin Nutr. 1980;33(1):27-39.

12. Bergström J, Heimbürger $\mathrm{O}$, Lindholm B. Calculation of the protein equivalent of total nitrogen appearance from urea appearance. Which formulas should be used? Perit Dial Int. 1998;18(5):467-473.

13. Elmadfa I, MajchrzakD, Rust P, GenserD. The thiamine status of adult humans depends on carbohydrate intake. Int J Vitam Nutr Res. 2001;71(4):217-221.

14. Weber W, Nitz M, Looby M. Nonlinear kinetics of the thiamine cation in humans: Saturation of nonrenal clearance and tubular reabsorption. J Pharmacokinet Biopharm. 1990;18(6):501-523. 
15. Jankowska M, Szupryczyńska N, Dębska-Ślizień A, et al. Dietary intake of vitamins in different options of treatment in chronic kidney disease: Is there a deficiency? Transplant Proc. 2016;48(5):1427-1430.

16. Bukhari FJ, Moradi H, Gollapudi P, Ju Kim H, Vaziri ND, Said HM. Effect of chronic kidney disease on the expression of thiamin and folic acid transporters. Nephrol Dial Transplant. 2011;26(7):2137-2144. 